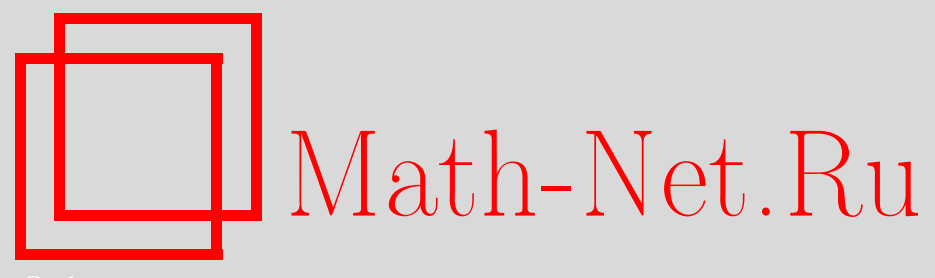

Б. Стаматович, О распознавании лабиринтов автоматами, Дискрет. матем., 2000, том 12, выпуск 2, 51-65

DOI: https://doi.org/10.4213/dm335

Использование Общероссийского математического портала Math-Net.Ru подразумевает, что вы прочитали и согласны с пользовательским соглашением http://www . mathnet.ru/rus/agreement

Параметры загрузки:

IP: 54.174 .149 .18

26 апреля 2023 г., 15:26:41 


\title{
О распознавании лабиринтов автоматами
}

\author{
(C) 2000 г. $\quad$ Б. Стаматович
}

\begin{abstract}
Изучается проблема существования автоматов, распознающих некоторые прямоугольные лабиринты. Доказывается отсутствие распознающего автомата для простых прямоугольных лабиринтов, что не имеет места для мозаичных лабиринтов. Приведен пример бесконечного класса $\pi$-лабиринтов, который не распознается автоматом.
\end{abstract}

\section{1. Основные понятия и результаты}

Основные понятия и обозначения из теории автоматов и теории графов, которыми мы пользуемся, совпадают с принятыми, соответственно, в [2, 3] и [4], и здесь не определяются.

Пусть $X_{\alpha}, \alpha \in I$, - некоторое множество. Тогда через $\operatorname{Pr}_{\alpha}$ обозначим отображение проектирования произведения $\prod_{\beta \in I} X_{\beta}$ на $\alpha$-й сомножитель $X_{\alpha}$.

Пусть $L=(V, E)$ - некоторый граф, где $V$ - множество его вершин и $E-$ множество его дуг. Множество вершин $V$ и множество дуг $E$ графа $L$ будем обозначать через $V(L)$ и $E(L)$.

Пусть $Y$ - некоторое множество. Если задана некоторая функция $f: E \rightarrow Y$, то пара $(L, f)$ называется нагруженным графом, множество $Y$ - множеством меток, а $f$ - разметкой (дуг) графа $L$. Элемент $f(e) \in Y$, где $e \in E$, называется меткой дуги $e$ в нагруженном графе $(L, f)$.

Если в графе $L=(V, E)$ вместе с дугой $\left(v_{1}, v_{2}\right)$ содержится дуга $\left(v_{2}, v_{1}\right)$, то называем эту пару ребром и обозначаем $\left\langle v_{1}, v_{2}\right\rangle$. Граф называется симметрическим, если при $\left(v_{1}, v_{2}\right) \in E$ имеет место $\left\langle v_{1}, v_{2}\right\rangle \subseteq E$.

Обозначим через $D$ множество векторов $\{e, n, w, s\}$, где $e$ и $n-$ соответственно базисные единичные векторы двумерного Евклидова пространства, а векторы $w$ и $s$ равны, соответственно, этим базисным векторам с противоположным направлением. Будем писать $e^{-1}=w, n^{-1}=s, w^{-1}=e$ и $s^{-1}=n$.

Нагруженный связный симметрический граф $(L, f)(L=(V, E), f: E \rightarrow Y)$ без кратных дуг и петель называется 2-лабиринтом, или в последующем просто лабиринтом, если выполнены следующие условия:

(1) $|V| \geqslant 2$ и $Y=D$,

(2) для любых $x, y \in E, x \neq y$, если $\operatorname{Pr}_{1}(x)=\operatorname{Pr}_{1}(y)$,то $f(x) \neq f(y)$,

(3) для любых $u, v \in V$, если $(u, v) \in E$, то $f((u, v))=(f((v, u)))^{-1}$. 
В последующем в обозначении лабиринта $(L, f)$ будем опускать $f$, считая, что в любом конкретном случае $f$ задана. Часто вместо $L$ будем писать $(L ; u)$ или $L_{u}$, $u \in V$, если в $L$ отмечена одна вершина $u$, при этом она называется входом или началом лабиринта $L$, а лабиринт $L$ инициальным.

Через $|x|_{L}$ обозначим значение $f$ на дуге $x$ лабиринта $L$. Если из контекста ясно, о каком лабиринте идет речь, то вместо $|x|_{L}$ будем писать $|x|$. Пусть $v-$ вершина лабиринта $L=(V, E)$. Множество меток $\left\{|x| \mid x \in E_{v}\right\}$, где $E_{v}=$ $\left\{x \in E \mid \operatorname{Pr}_{1}(x)=v\right\}$, обозначим через $[v]_{L}$.

Пусть $M$ и $N, M \neq N$, - некоторые точки плоскости и $\overline{M N}=\alpha_{1} e+\alpha_{2} n, e$ и $n-$ единичные координатные векторы прямоугольной системы координат на плоскости. Будем говорить, что отрезок $\overline{M N}$ идет в направлении $e$, если $\alpha_{1}>0$ и $\alpha_{2}=0$, в направлении $n$, если $\alpha_{1}=0$ и $\alpha_{2}>0$, в направлении $w$, если $\alpha_{1}<0$ и $\alpha_{2}=0$ и в направлении $s$, если $\alpha_{1}=0$ и $\alpha_{2}<0$. Множество $T$ отрезков на плоскости называется конфигурацией, если любые два разных отрезка из этого множества могут иметь не больше одной общей точки, причем, если она у них есть, то она обязательно является концевой для обоих отрезков.

Лабиринт $L=(V, E)$, где $V \subseteq R^{2}$, называем прямоугольным лабиринтом, если для любых $u, v \in V$ из $(u, v) \in E$ отрезок $\overline{u v}$ идет в направлении $|(u, v)|$, а множество отрезков $T=\{\overline{u v} \mid(u, v) \in E\}$ является конфигурацией. Фигура $\bar{L}_{(u, v) \in E(L)} \overline{u v}$ в $R^{2}$ называется реализацией прямоугольного лабиринта $L$.

Пусть $Z^{2}$ - целочисленная решетка на плоскости. Проведем через вершины $Z^{2}$ все возможные прямые, параллельные осям координат. Полученная фигура является реализацией прямоугольного лабиринта, который обозначим через $\mathbf{Z}^{2}$. Под мозаичным лабиринтом будем понимать любую связную часть (нагруженную) лабиринта $\mathbf{Z}^{2}$. Под шахматным лабиринтом будем понимать любой связный подграф (нагруженный) лабиринта $\mathbf{Z}^{2}$.

Назовем лабиринты $L_{1}$ и $L_{2}$ слабо изоморфными, если существует биекция $g: V\left(L_{1}\right) \rightarrow V\left(L_{2}\right)$ такая, что $(u, v) \in E\left(L_{1}\right)$ тогда и только тогда, когда $(g(u), g(v)) \in$ $E\left(L_{2}\right)$, причем, если один из них с отмеченным входом, то таким является и другой и выполняется соотношение $g\left(v_{0}^{1}\right)=v_{0}^{2}$, где $v_{0}^{1}$ - вход лабиринта $L_{1}$, а $v_{0}^{2}-$ вход лабиринта $L_{2}$. Если еще $|(u, v)|_{L_{1}}=|(g(u), g(v))|_{L_{2}}$ для любой дуги $(u, v) \in E\left(L_{1}\right)$, то $L_{1}$ и $L_{2}$ называются изоморфными. Мы не различаем изоморфные лабиринты и в таком случае пишем, что $L_{1}=L_{2}$.

Введем функцию расстояния $d$ в $Z^{2}$ следующим способом. Для любых двух точек $u, v \in Z^{2}$ положим

$$
d(u, v)=\left(\left(\operatorname{Pr}_{1}(u)-\operatorname{Pr}_{1}(v)\right)^{2}+\left(\operatorname{Pr}_{2}(u)-\operatorname{Pr}_{2}(v)\right)^{2}\right)^{1 / 2} .
$$

Для любого конечного множества $V \subseteq Z^{2}$ обозначим

$$
\operatorname{diam}(V)=\max \{d(u, v) \mid u, v \in V\} .
$$

Пусть $a=\left(a_{1}, a_{2}\right)$ и $b=\left(b_{1}, b_{2}\right)$ - произвольные элементы множества $Z^{2}$. Говорим, что $a$ и $b$ (слабо) соседние, если $(d(a, b)<2) d(a, b)=1$. Последовательность

$$
a=p_{0}, p_{1}, \ldots, p_{m}=b
$$

в $Z^{2}$ называется (слабой) цепью, связывающей точку $a$ и точку $b$, если точки $p_{i-1}$ и $p_{i}$ являются (слабо) соседними для любого $i, 1 \leqslant i \leqslant m$. Множество $V, V \subseteq Z^{2}$, называется (слабо) связным, если для любых $a, b \in V$ существует (слабая) цепь в $V$, 
связывающая их. Компонентой (слабой) связности множества $V$ называется любое максимальное (слабо) связное подмножество множества $V$.

Любое отображение $c: Z^{2} \rightarrow E^{2}, E^{2}=\{1,0\}$, такое, что $P_{c}=c^{-1}(\{1\})$ является связным множеством, называется $\pi$-лабиринтом. Если $p_{0}-$ произвольная точка в $P_{c}$, тогда пара $\left(c, p_{0}\right)$ называется $\pi$-лабиринтом с началом $p_{0}$. Если множество $P_{c}$ является (конечным) бесконечным, то $\pi$-лабиринт называется (конечным) бесконечным. Далее под $\pi$-лабиринтом будем понимать конечный $\pi$-лабиринт. Дырой $\pi$-лабиринта $c$ называется произвольная компонента слабой связности множества $Z^{2} \backslash P_{c}$.

Автомат $\mathbf{A}_{q_{0}}=\left(A, Q, B, \varphi, \psi, q_{0}\right)$ называется допустимым, если $A$ - множество всех непустых подмножеств множества $D=\{e, n, w, s\}, B=D \cup\{0\}$ и $\psi(q, a) \in a \cup\{0\}$ для всех $q \in Q$ и $a \in A$. В дальнейшем предполагаем, что все автоматы являются допустимыми. Поведением автомата $\mathbf{A}_{q_{0}}$ в лабиринте $L_{v_{0}}$ называем последовательность

$$
\pi\left(\mathbf{A}_{q_{0}} ; L_{v_{0}}\right)=\left(q_{0}, v_{0}\right),\left(q_{1}, v_{1}\right), \ldots,
$$

где $\left(v_{i}, v_{i+1}\right) \in E\left(L_{v_{0}}\right)$ или

$$
v_{i}=v_{i+1}, \quad q_{i+1}=\varphi\left(q_{i},\left[v_{i}\right]_{L}\right), \quad \psi\left(q_{i},\left[v_{i}\right]_{L}\right)=\left|v_{i}, v_{i+1}\right|, \quad i=0,1, \ldots
$$

Через $\pi\left(\mathbf{A}_{q_{0}} ; L_{v_{0}} ; s\right)$ обозначим начальный отрезок длины $s$ последовательности $\pi\left(\mathbf{A}_{q_{0}} ; L_{v_{0}}\right)$. Последовательность $\left|\left(v_{0}, v_{1}\right)\right|,\left|\left(v_{1}, v_{2}\right)\right|, \ldots$ обозначим через $\operatorname{Tr}\left(\mathbf{A}_{q_{0}}, L_{v_{0}}\right)$. Пусть

$$
\operatorname{Int}\left(\mathbf{A}_{q_{0}}, L_{v_{0}}\right)=\bigcup_{i=1}^{\infty}\left\{v_{i}\right\} .
$$

Если $\operatorname{Int}\left(\mathbf{A}_{q_{0}}, L_{v_{0}}\right)=V\left(L_{0}\right)$, то говорим, что автомат $\mathbf{A}_{q_{0}}$ обходит лабиринт $L_{v_{0}}$. Если для любого $u \in V(L)$ автомат $\mathbf{A}_{q_{0}}$ обходит лабиринт $L_{u}$, то говорим, что автомат $\mathbf{A}_{q_{0}}$ сильно обходит лабиринт $L$.

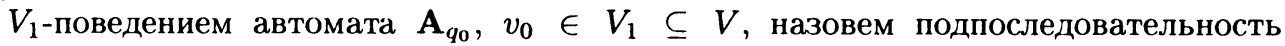
$\left(q_{i_{0}}, v_{i_{0}}\right),\left(q_{i_{1}}, v_{i_{1}}\right), \ldots$ поведения $\pi\left(\mathbf{A}_{q_{0}} ; L_{v_{0}}\right)$, которая получается из $\pi\left(\mathbf{A}_{q_{0}} ; L_{v_{0}}\right)$, когда из него выбрасываются все пары $\left(q_{i}, v_{i}\right)$, для которых $v_{i} \notin V_{1}$. Определим для любого $V_{1} \subseteq V$ значения

$$
\operatorname{st}\left(\pi, V_{1}\right), \quad \operatorname{pl}\left(\pi, V_{1}\right), \quad \operatorname{dr}\left(\pi, V_{1}\right), \quad \operatorname{tm}\left(\pi, V_{1}\right),
$$

где $\pi=\pi\left(\mathbf{A}_{q_{0}} ; L_{v_{0}}\right)$, следующим образом. Если существует такое $t, t>0$, что $v_{t} \in V_{1}$ и для любого $t^{\prime}, 0<t^{\prime}<t, v_{t^{\prime}} \notin V_{1}$, то

$$
\begin{aligned}
\operatorname{st}\left(\pi, V_{1}\right) & =q_{t}, & \operatorname{pl}\left(\pi, V_{1}\right) & =v_{t}, \\
\operatorname{dr}\left(\pi, V_{1}\right) & =\psi\left(q_{t},\left[v_{t}\right]_{L}\right), & \operatorname{tm}\left(\pi, V_{1}\right) & =t,
\end{aligned}
$$

в противном случае $\operatorname{st}\left(\pi, V_{1}\right), \operatorname{pl}\left(\pi, V_{1}\right), \operatorname{dr}\left(\pi, V_{1}\right), \operatorname{tm}\left(\pi, V_{1}\right)$ не определены.

Пусть $Q_{F}=\left\{q_{F_{0}}, q_{F_{1}}\right\}$ и $Q_{F} \subseteq Q\left(\mathbf{A}_{q_{0}}\right)$. Будем говорить, что автомат $\mathbf{A}_{q_{0}}$ распознает лабиринт $L_{v}$, если при его запуске в лабиринт $L_{v}$ в итоге происходит переход в заключительное состояние $q_{F_{1}}$, а при его запуске в лабиринт $L_{v^{\prime}}^{\prime} \neq L_{v}$ происходит переход в заключительное состояние $q_{F_{0}}$. Пусть $\mathbf{C}$ - класс инициальных лабиринтов. Говорим, что автомат $\mathbf{A}_{q_{0}}$ распознает класс $\mathbf{C}$, если при его запуске в любой лабиринт $L_{v}$ происходит переход в заключительное состояние $q_{F_{1}}$ тогда только тогда, когда $L_{v} \in \mathbf{C}$ и для любого лабиринта $L_{v} \notin \mathbf{C}$ происходит переход в заключительное состояние $q_{F_{0}}$. 
Пусть $c$ - произвольный $\pi$-лабиринт. Рассмотрим граф $G_{c}=\left(P_{c}, X_{c}\right)$, у которого $P_{c}-$ множество вершин, $X_{c}-$ множество дуг и $\left\langle p_{1}, p_{2}\right\rangle \in X_{c}$ тогда и только тогда, когда $p_{1}$ и $p_{2}$ из $P_{c}-$ соседние точки.

Пусть $\mathbf{V}=\left(p_{1}, p_{2}, \ldots, p_{k-1}\right)-$ упорядоченный набор различных ненулевых элементов из $Z^{2}$ и $\mathbf{A}=\left(A, Q, B, \varphi, \psi, q_{0}\right)$ - произвольный инициальный автомат такой, что $B=V^{\prime}, V^{\prime} \subseteq\left\{0, p_{1}, p_{2}, \ldots, p_{k-1}\right\}$ и $A=\left(E^{2}\right)^{k}$. Пару $(\mathbf{A}, \mathbf{V})$ назовем пешкой, $\mathbf{V}$ - полем зрения этой пешки. Пешка $(\mathbf{A}, \mathbf{V})$ называется регулярной, если $\psi$ такое, что для произвольного $q \in Q$ и

$$
a=\left(a_{1}, \ldots, a_{k}\right)=\left(1, a_{2}, \ldots, a_{k}\right) \in\left(E^{2}\right)^{k}
$$

из того, что $\psi(q, a)=p_{i}$ для некоторого $i, 0 \leqslant i \leqslant k-1$, следует, что $a_{i+1}=1, p_{0}=0$.

Пусть даны регулярная пешка $(\mathbf{A}, \mathbf{V})$ и $\pi$-лабиринт $\left(c, p_{0}\right)$,

$$
\mathbf{V}=\left(p_{1}, p_{2}, \ldots, p_{k-1}\right), \quad \mathbf{A}=\left(A, Q, B, \varphi, \psi, q_{0}\right) .
$$

Набор $\mathbf{V}$ определяет для каждого $z \in Z^{2}$ набор

$$
\mathbf{V}(z)=\left(z, z+p_{1}, z+p_{2}, \ldots, z+p_{k-1}\right) \text {. }
$$

Тогда поведением пешки $(\mathbf{A}, \mathbf{V})$ в $\pi$-лабиринте $\left(c, p_{0}\right)$ называется последовательность

$$
\pi\left(\mathbf{A}, \mathbf{V} ; c, p_{0}\right):\left(z_{0}, q_{0}, a_{0}, b_{0}\right),\left(z_{1}, q_{1}, a_{1}, b_{1}\right), \ldots,
$$

где

$$
\begin{gathered}
z_{0}=p_{0}, \quad z_{i+1}=z_{i}+b_{i}, \quad q_{i+1}=\varphi\left(q_{i}, a_{i}\right), \\
a_{i}=c\left[\mathbf{V}\left(z_{i}\right)\right]=\left(c\left(z_{i}\right), c\left(z_{i}+p_{1}\right), \ldots, c\left(z+p_{k-1}\right)\right), \quad b_{i}=\psi\left(q_{i}, a_{i}\right) .
\end{gathered}
$$

Ясно, что если пешка $(\mathbf{A}, \mathbf{V})$ регулярна, то $z_{t} \in P_{c}$ для любого $t, t=0,1, \ldots$

В дальнейшем будем рассматривать только регулярные пешки, для которых

$$
\mathbf{V}=((1,-1),(1,0),(1,1),(0,-1),(0,1),(-1,-1),(-1,0),(-1,1))
$$

и $B=D \cup\{0\}$.

Пусть $L=\left(V, E ; v_{0}\right)$ - некоторый инициальный лабиринт. Если для некоторого взаимно однозначного отображения $\mu$ множества $V$ в плоскость существует такой прямоугольный лабиринт вида $L_{1}=\left(\mu(V), E_{1} ; \mu\left(v_{0}\right)\right)$, что лабиринты $L$ и $L_{1}$ являются изоморфными, причем $\mu$ определяет этот изоморфизм, то говорим, что лабиринт $L$ вложим. В случае, когда $L$ вложим, отображение $\mu$ называется укладкой. Ясно, что если при фиксированном $\mu$ существует $L_{1}$, то он единствен. Исходя из этого, под укладкой будем иногда понимать $L_{1}$ или даже $\bar{L}_{1}$. Далее ясно, что если лабиринт $L$ является деревом, то он вложим.

Пусть лабиринт $L=(V, E)$ такой, что

$$
V=V_{x} \cup V_{y}, \quad V x \cap V_{y}=\varnothing, \quad\left|V_{x}\right|=\left|V_{y}\right|=k,
$$

где $k \geqslant 2$ и $V_{x}=\left\{x_{1}, x_{2}, \ldots, x_{k}\right\}, V_{y}=\left\{y_{1}, y_{2}, \ldots, y_{k}\right\}$. Лабиринт $L$ называется $w$-лестницей, $w \in\{e, n\}$, если существует укладка $\mu$, такая что $\operatorname{Pr}_{m}\left(\mu\left(x_{i}\right)\right)=$ $\operatorname{Pr}_{m}\left(\mu\left(x_{j}\right)\right), \operatorname{Pr}_{m}\left(\mu\left(y_{i}\right)\right)=\operatorname{Pr}_{m}\left(\mu\left(y_{j}\right)\right), \operatorname{Pr}_{n}\left(\mu\left(x_{s}\right)\right)=\operatorname{Pr}_{n}\left(\mu\left(y_{s}\right)\right), i \neq j, i, j, s \in$ 
$\{1, \ldots, k\}$, и если $\operatorname{Pr}_{n}\left(\mu\left(x_{i_{1}}\right)\right)<\operatorname{Pr}_{n}\left(\mu\left(x_{i_{2}}\right)\right)<\ldots<\operatorname{Pr}_{n}\left(\mu\left(x_{i_{k}}\right)\right), i_{j} \in\{1,2, \ldots, k\}$, TO

$$
\begin{aligned}
E=\left\{\left\langle x_{i_{j-1}}, x_{i_{j}}\right\rangle,\left\langle x_{i_{j}}, x_{i_{j+1}}\right\rangle \mid j=2, \ldots, k-1\right\} \\
\cup\left\{\left\langle y_{i_{j-1}}, y_{i_{j}}\right\rangle,\left\langle y_{i_{j}}, x_{y_{j+1}}\right\rangle \mid j=2, \ldots, k-1\right\} \cup\left\{\left\langle x_{i}, y_{i}\right\rangle \mid i=1, \ldots, k\right\},
\end{aligned}
$$

где $(m, n)=(1,2)$, если $w=n$, и $(m, n)=(2,1)$, если $w=e$ (лабиринт $L$ называем лестницей, если он $е$-лестница или $n$-лестница).

Пусть $L_{1}=\left(V_{1}, E_{1}\right)$ и $L_{2}=\left(V_{2}, E_{2}\right), V_{1} \cap V_{2}=\varnothing$, суть произвольные лабиринты, $x_{1} \in V_{1}, x_{2} \in V_{2}$ и $\left[x_{1}\right]_{L_{1}} \cap\left[x_{2}\right]_{L_{2}}=\varnothing$. Пусть $V_{2}^{\prime}=V_{2} \backslash\left\{x_{2}\right\}$. Пусть лабиринт $L\left(x_{1}\right)=\left(V_{2}\left(x_{1}\right), E_{2}\left(x_{1}\right)\right)$, где $V_{2}\left(x_{1}\right)=\left\{x_{1}\right\} \cup\left\{x_{1}\right\} \times V_{2}^{\prime}$, такой, что отображение

$$
i: V_{2} \rightarrow V_{2}\left(x_{1}\right), \quad i\left(x_{2}\right)=x_{1}, \quad i(x)=\left(x_{1}, x\right), \quad x \in V_{2}^{\prime},
$$

определяет изоморфизм лабиринтов $L_{2}$ и $L\left(x_{1}\right)$. Тогда через

$$
L_{1} \underset{x_{1}, x_{2}}{+} L_{2}
$$

обозначим лабиринт

$$
\left(V_{1} \cup V_{2}\left(x_{1}\right), E_{1} \cup E_{2}\left(x_{1}\right)\right),
$$

причем, если $u \in E_{1}$, то $|u|=|u|_{L_{1}}$, а если $u \in E_{2}\left(x_{1}\right)$, то $|u|=|u|_{L_{2}}$. Если в $L_{1}$ отмечен вход, то в лабиринте $L_{1} \underset{x_{1}, x_{2}}{+} L_{2}$ отмечен тот же самый вход. Скажем, что лабиринт $L_{1} \underset{x_{1}, x_{2}}{+} L_{2}$ получаем склеиванием вершин $x_{1}$ и $x_{2}$ лабиринтов $L_{1}$ и $L_{2}$. В [2] показано, что если $L_{1}, L_{2}$ суть вложимые лабиринты такие, что $L_{2}$ является деревом, то лабиринт $L_{1}+\underset{x_{1}, x_{2}}{+} L_{2}$ вложим.

Пусть $\mathscr{S}$ есть множество лабиринтов $L=(V, E)$ таких, что $L-$ лестница или существуют $x_{1}, \ldots, x_{s} \in V$, лабиринты деревья $L_{1}, \ldots, L_{s}$ и лабиринт-лестница $L^{\prime}$ такие, что

$$
L=L^{\prime} \underset{x_{1}, x^{1}}{+} L_{1} \underset{x_{2}, x^{2}}{+} \cdots \underset{x_{s}, x^{s}}{+} L_{s}
$$

где $x_{1}, \ldots, x_{s} \in V\left(L^{\prime}\right), x^{i} \in V\left(L_{i}\right), i \in\{1,2, \ldots, s\}$.

Скажем, что лабиринт $L$ является $\varepsilon$-вложимым, если существует укладка $\mu$ лабиринта $L$ такая, что $\operatorname{diam}(\mu(V(L)))<\varepsilon$. Ясно, что если лабиринт $L$ есть дерево или лестница, то $L$ является $\varepsilon$-вложимым для каждого $\varepsilon>0$.

Теорема 1. Eсли лабиринт $L=(V, E) \in \mathscr{S}$, то для каждого $v \in V$ не существует автомат, которьй распознает лабиринт $L_{v}$.

Теорема 2. Если $L=(V, E)$ - мозаичнљй лабиринт, то существует автомат, который распознает класс $\left\{L_{v} \mid v \in V\right\}$.

Пусть $\mathbf{P}-$ множество таких $K \subseteq Z^{2}$, что $K$ - связное конечное множество, для которого множество $Z^{2} \backslash K$ связно.

Если множество $K \in \mathbf{P}$, то границей $\partial K$ множества $K$ будем называть множество точек $z \in K$, для которых существует точка $z^{\prime \prime}$ в $Z^{2} \backslash K$ такая, что $z$ и $z^{\prime \prime}$ 


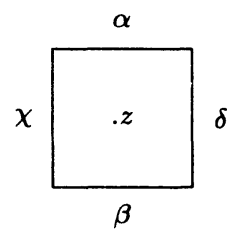

Рис. 1.

слабо соседние. Вокруг каждой точки $z=\left(z_{1}, z_{2}\right) \in \partial K$ рассмотрим квадрат $k v_{z} \mathrm{c}$ единичной длиной сторон (см. рис. 1). Его стороны обозначим через $\alpha, \beta, \chi, \delta$ :

$$
\begin{aligned}
& \alpha=\left\{x=\left(x_{1}, x_{2}\right) \in \mathbf{R}^{2} \mid z_{1}-1 / 2 \leqslant x_{1} \leqslant z_{1}+1 / 2, x_{2}=z_{2}+1 / 2\right\}, \\
& \beta=\left\{x=\left(x_{1}, x_{2}\right) \in \mathbf{R}^{2} \mid z_{1}-1 / 2 \leqslant x_{1} \leqslant z_{1}+1 / 2, x_{2}=z_{2}-1 / 2\right\}, \\
& \chi=\left\{x=\left(x_{1}, x_{2}\right) \in \mathbf{R}^{2} \mid x_{1}=z_{1}-1 / 2, z_{2}-1 / 2 \leqslant x_{2} \leqslant z_{2}+1 / 2\right\}, \\
& \delta=\left\{x=\left(x_{1}, x_{2}\right) \in \mathbf{R}^{2} \mid x_{1}=z_{1}+1 / 2, z_{2}-1 / 2 \leqslant x_{2} \leqslant z_{2}+1 / 2\right\} .
\end{aligned}
$$

Стороны $\alpha, \beta, \chi, \delta$ квадрата $k v_{z}, z \in K$, имеют такое свойство: сторона находится между точками множества $K$ и множества $\mathbf{Z}^{2} \backslash K$, если точки $\left(z_{1}, z_{2}+1\right),\left(z_{1}, z_{2}-1\right)$, $\left(z_{1}-1, z_{2}\right),\left(z_{1}+1, z_{2}\right)$ не принадлежат множеству $K$, соответственно. Пусть $\mathrm{st}_{z}$ множество сторон квадрата $k v_{z}$, для которых сторона находится между точками множества $K$ и множества $\mathbf{Z}^{2} \backslash K$. Фигура

$$
F_{K}=\bigcup_{z \in \partial K} \mathrm{st}_{z}
$$

представляет собой прямоугольный полигон.

Пусть $K \subset \mathbf{Z}^{2}$ - конечное связное множество. Самая нижняя и самая правая точка (НП) множества $K$ есть точка $z=\left(z_{1}, z_{2}\right) \in K$ такая, что для каждого $a=$ $\left(a_{1}, a_{2}\right) \in K$ или $z_{2}<a_{2}$, или, если $z_{2}=a_{2}$, то $z_{1}>a_{1}$. Самая нижняя и самая левая точка (НЛ) множества $K$ есть точка $z=\left(z_{1}, z_{2}\right) \in K$ такая, что для каждого $a=\left(a_{1}, a_{2}\right) \in K$ или $z_{2}<a_{2}$, или, если $z_{2}=a_{2}$, то $z_{1}<a_{1}$. Самая высокая и самая правая точка (ВП) множества $K$ есть точка $z=\left(z_{1}, z_{2}\right) \in K$ такая, что для каждого $a=\left(a_{1}, a_{2}\right) \in K$ или $z_{2}>a_{2}$, или, если $z_{2}=a_{2}$, то $z_{1}>a_{1}$. Самая высокая и самая левая точка (ВЛ) множества $K$ есть точка $z=\left(z_{1}, z_{2}\right) \in K$ такая, что для каждого $a=\left(a_{1}, a_{2}\right) \in K$ или $z_{2}>a_{2}$, или, если $z_{2}=a_{2}$, то $z_{1}<a_{1}$.

Пусть $(S)^{*}$ - множество всех слов $\alpha=\alpha(1) \alpha(2) \ldots \alpha(k), k \geqslant 4$, над алфавитом $S=\{-1,1\}$. Определим отображение $f: \mathbf{P} \rightarrow(S)^{*}$ следующим образом. Пусть $P \in$ P. Обходя полигон $F_{P}$ в положительном направлении, начиная от самой нижней и самой правой точки, пронумерируем вершины полигона $F_{P}$, сопоставляя им -1 , если угол в вершине равен $\pi / 2$, и 1 , если он равен $-\pi / 2$. 


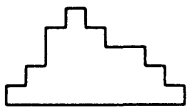

$\left(-1(-1,1)^{4}-1-1(1,-1)^{3}-1\right)$

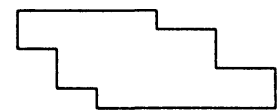

$\left(-1(-1,1)^{2}-1-1(-1,1)^{2}-1\right)$

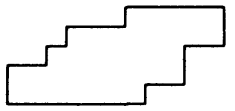

$\left(-1(1,-1)^{2}-1-1(1,-1)^{3}-1\right)$

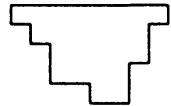

$\left(-1(1,-1)^{2}-1-1(-1,1)^{3}-1\right)$

Pис. 2.

Определим следующие семейства множеств (рис. 2):

$$
\begin{aligned}
& \Phi_{1}=\left\{P \in \mathbf{P} \mid\|P\| \geqslant 2, f(P)=\left(-1(-1,1)^{n}-1-1(1,-1)^{k}-1\right), k, n \geqslant 0\right\}, \\
& \Phi_{2}=\left\{P \in \mathbf{P} \mid\|P\| \geqslant 2, f(P)=\left(-1(1,-1)^{n}-1-1(1,-1)^{k}-1\right), k, n \geqslant 0\right\}, \\
& \Phi_{3}=\left\{P \in \mathbf{P} \mid\|P\| \geqslant 2, f(P)=\left(-1(-1,1)^{n}-1-1(-1,1)^{k}-1\right), k, n \geqslant 0\right\}, \\
& \Phi_{4}=\left\{P \in \mathbf{P} \mid\|P\| \geqslant 2, f(P)=\left(-1(1,-1)^{n}-1-1(-1,1)^{k}-1\right), k, n \geqslant 0\right\},
\end{aligned}
$$

где $(a, b)^{n}$ обозначает произведение $(a b)(a b) \ldots(a b)$, состоящее из $n$ сомножителей, $n \in N$.

Если $z_{j}=\left(x_{j}, y_{j}\right) \in \mathbf{Z}^{2}, j=1,2,3,4$, такие, что $y_{2}=y_{3}, y_{1}=y_{4}$ и $y_{1} \leqslant y_{2}$, то пусть $A_{\Phi_{1}}^{z_{1}, z_{2}, z_{3}, z_{4}}-$ множество таких $K \in \Phi_{i}$, что $z_{1}, z_{2}, z_{3}, z_{4}-$ НП, ВП, ВЛ, НЛ точки множества $K$, соответственно, $i=1, \ldots, 4$.

Пусть $z_{i}=\left(x_{i}, y_{i}\right) \in Z^{2}, i=1,2, \ldots, 16$, имеют следующее свойство 0 :

$$
\begin{array}{ll}
y_{9}=y_{16}=y_{11}=y_{2}, & \\
x_{9} \leqslant x_{16}<x_{11}-1, & x_{11} \leqslant x_{2}, \\
y_{4}>y_{3}>y_{2}, & \\
x_{7} \leqslant x_{14}<x_{13}-1, & x_{13} \leqslant x_{4}, \\
y_{7}>y_{8}>y_{9} . &
\end{array}
$$

Тогда $K_{0}^{\left\{z_{i}, i=1, \ldots, 16\right\}}$ есть множество таких $K \in \mathbf{P}$, что

$$
K=K_{1}^{0} \cup K_{2}^{0} \cup K_{3}^{0} \cup K_{4}^{0} \cup K_{5}^{0} \cup K_{6}^{0}
$$

где

$$
\begin{array}{ll}
K_{1}^{0} \in A_{\Phi_{4}}^{z_{1}, z_{2}, z_{9}, z_{10}}, & K_{2}^{0} \in A_{\Phi_{2}}^{z_{2}, z_{3}, z_{12}, z_{11}}, \\
K_{3}^{0} \in A_{\Phi_{3}}^{z_{3}, z_{4}, z_{13}, z_{12}}, & K_{4}^{0} \in A_{\Phi_{1}}^{z_{4}, z_{5}, z_{6}, z_{7}}, \\
K_{5}^{0} \in A_{\Phi_{2}}^{z_{15}, z_{14}, z_{7}, z_{8}}, & K_{6}^{0} \in A_{\Phi_{3}}^{z_{16}, z_{15}, z_{8}, z_{9}},
\end{array}
$$




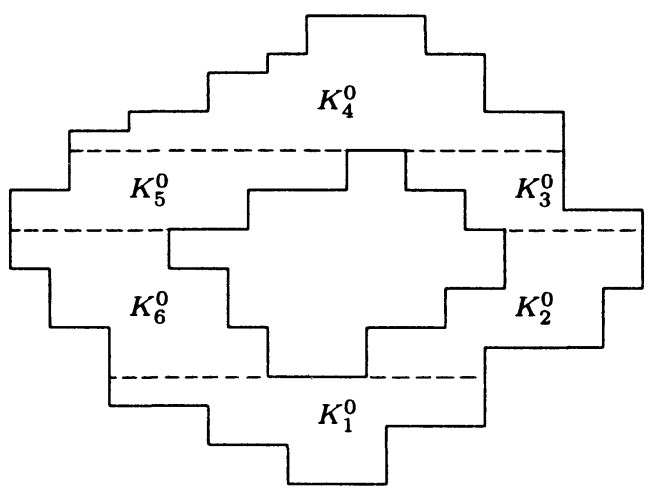

Рис. 3.

и выполнены условия

$$
\begin{aligned}
\left(x_{1}=x_{10}\right) & \Rightarrow\left(z_{1}+(1,1) \in K_{1}^{0} \wedge z_{1}+(-1,1) \in K_{1}^{0}\right), \\
\left(z_{3}+(0,1) \notin K_{3}^{0} \wedge z_{3}+(0,-1) \notin K_{2}^{0}\right) & \Rightarrow\left(z_{3}+(-1,1) \in K_{3}^{0} \vee z_{3}+(-1,-1) \in K_{2}^{0}\right), \\
\left(x_{5}=x_{6}\right) & \Rightarrow\left(z_{5}+(1,-1) \in K_{4}^{0} \wedge z_{5}+(-1,-1) \in K_{4}^{0}\right), \\
\left(z_{8}+(0,1) \notin K_{5}^{0} \wedge z_{8}+(0,-1) \notin K_{6}^{0}\right) & \Rightarrow\left(z_{8}+(1,1) \in K_{5}^{0} \vee z_{8}+(1,-1) \in K_{6}^{0}\right) .
\end{aligned}
$$

Определим класс $\pi$-лабиринтов $\mathbf{C}_{0}$, полагая

$$
\begin{aligned}
\mathbf{C}_{0}=\left\{c: Z^{2} \rightarrow E^{2} \mid c^{-1}(\{1\})=\right. & K \in K_{0}^{\left\{z_{i}, \imath=1, \ldots, 16\right\}}, \\
& \left.z_{i} \in Z^{2}, i=1, \ldots, 16, \text { и выполняется условие } 0\right\}
\end{aligned}
$$

Теорема 3. Не существует пешки, которая распознает класс $\pi$-лабиринтов

$$
\begin{aligned}
\left(\mathbf{C}_{0}, \mathrm{H \Pi}\right)=\left\{\left(c, p_{\mathrm{H}}\right) \mid c \in \mathbf{C}_{0},\right. \\
\left.p_{\mathrm{H} \Pi}-\text { самая нижняя и самая правая точка множества } c^{-1}(\{1\})\right\} .
\end{aligned}
$$

\section{2. Доказательство теоремы 1}

Предположим, что лабиринт $L=(V, E)$ есть $n$-лестница. Пусть автомат

$$
\mathbf{A} q_{0}=\left(A, Q, B, \varphi, \psi, q_{0}\right)
$$

распознает лабиринт $L=\left(V, E ; v_{0}\right)$ для некоторого $v_{0} \in V$. Если $v \in V$, то обозначим через $n m(v)$ число появлений вершины $v$ в последовательности $\pi\left(\mathbf{A}_{q_{0}} ; L_{v_{0}} ; t\right)$, где $t \geqslant 0$ такое, что $\varphi\left(q_{t},\left[v_{t}\right]\right)=q_{F_{1}}$. Пусть $n=\max \{n m(v) \mid v \in V\}$. Пусть $u, v \in V$ такие, что $[u]=\{e, s\}$ и $[v]=\{w, s\}(\langle u, v\rangle \in E)$. Пусть

$$
\begin{gathered}
L^{\prime}=\left(V^{\prime}, E^{\prime}\right), \quad V^{\prime}=V \cup\{x, y\} \\
E^{\prime}=\langle u, y\rangle \cup\langle v, x\rangle \cup E \backslash\langle u, v\rangle, \quad[(u, y)]=e, \quad[(v, x)]=w .
\end{gathered}
$$


Пусть

$$
L^{y}=L^{\prime} \backslash\{x\}, \quad L^{x}=L^{\prime} \backslash\{y\}
$$

и

$$
L_{1}=\left(\left(\left(L^{\prime} \underset{y, z^{1}}{+} L^{y_{k}^{1}}\right) \underset{y_{k}^{1}, z^{2}}{+} L^{y_{k}^{2}}\right) \underset{y_{k}^{2}, z^{3}}{+} \cdots \underset{y_{k}^{n}, z^{n+1}}{+} L^{y_{k}^{n+1}}\right),
$$

где лабиринт $L^{y_{k}^{2}}$ изоморфен лабиринту $L^{y}$ и получен переобозначением $f_{i}: V\left(L^{y}\right) \rightarrow$ $V\left(L^{y_{k}^{i}}\right)$, при этом $f_{i}(y)=y_{k}^{i}$ и $f_{i}(v)=z^{i}, 1 \leqslant i \leqslant n+1$.

Пусть

$$
\widetilde{L}=\left(\left(\left(L_{1} \underset{x, w^{1}}{+} L^{x_{k}^{1}}\right) \underset{x_{k}^{1}, w^{2}}{+} L^{x_{k}^{2}}\right) \underset{x_{k}^{2}, w^{3}}{+} \cdots \underset{x_{k}^{n}, w^{n+1}}{+} L^{x_{k}^{n+1}}\right),
$$

где лабиринт $L^{x_{k}^{2}}$ изоморфен лабиринту $L^{x}$ и получен переобозначением $g_{i}: V\left(L^{x}\right) \rightarrow$ $V\left(L^{x_{k}^{2}}\right)$, при этом $g_{i}(y)=x_{k}^{i}$ и $g_{i}(v)=w^{i}, 1 \leqslant i \leqslant n+1$.

Требуется показать, что лабиринт $\widetilde{L}$ вложим.

Пусть $\mu-$ укладка лабиринта $L$ такая, что

$$
\begin{gathered}
\mu(V(L))=\left\{x_{1}, \ldots, x_{k}, y_{1}, \ldots, y_{k}\right\} \\
\operatorname{Pr}_{1}\left(x_{i}\right)=\operatorname{Pr}_{1}\left(x_{j}\right), \operatorname{Pr}_{1}\left(y_{i}\right)=\operatorname{Pr}_{1}\left(y_{j}\right), \operatorname{Pr}_{2}\left(x_{s}\right)=\operatorname{Pr}_{2}\left(y_{s}\right), i \neq j, i, j, s \in\{1, \ldots, k\},
\end{gathered}
$$

и

$$
\operatorname{Pr}_{2}\left(x_{1}\right)<\operatorname{Pr}_{2}\left(x_{2}\right)<\ldots<\operatorname{Pr}_{2}\left(x_{k}\right) .
$$

Предположим также, что $\operatorname{Pr}_{1}\left(y_{1}\right)>\operatorname{Pr}_{1}\left(x_{1}\right)$ (см. рис. 4a).

Пусть $x, y \in \mathbf{R}^{2}$ такие, что

$$
\operatorname{Pr}_{2}\left(y_{k}\right)=\operatorname{Pr}_{2}(y)=\operatorname{Pr}_{2}(x)=\operatorname{Pr}_{2}\left(x_{k}\right), \quad \operatorname{Pr}_{1}\left(x_{k}\right)<\operatorname{Pr}_{1}(y)<\operatorname{Pr}_{1}(x)<\operatorname{Pr}_{1}\left(y_{k}\right) .
$$

Удалим отрезок $\overline{y x}$. Таким способом мы определили укладку лабиринта $L^{\prime}$ (см. рис. 4б).

Пусть $\mu^{\prime}-$ укладка лабиринта $L$ такая, что

$$
\mu^{\prime}(V(L))=\left\{x_{1}^{1}, \ldots, x_{k}^{1}, y_{1}^{1}, \ldots, y_{k}^{1}\right\}
$$

$$
\operatorname{Pr}_{1}\left(x_{i}^{1}\right)=\operatorname{Pr}_{1}\left(x_{j}^{1}\right), \operatorname{Pr}_{1}\left(y_{i}^{1}\right)=\operatorname{Pr}_{1}\left(y_{j}^{1}\right), \operatorname{Pr}_{2}\left(x_{s}^{1}\right)=\operatorname{Pr}_{2}\left(y_{s}^{1}\right), i \neq j, i, j, s \in\{1, \ldots, k\}
$$

и

$$
\operatorname{Pr}_{2}\left(x_{1}^{1}\right)<\operatorname{Pr}_{2}\left(x_{2}^{1}\right)<\ldots<\operatorname{Pr}_{2}\left(x_{k}^{1}\right) .
$$

Предположим, что $\operatorname{Pr}_{1}\left(y_{1}^{1}\right)>\operatorname{Pr}_{1}\left(x_{1}^{1}\right)$. Пусть $x \in \mathbf{R}^{2}$ такой, что

$$
\operatorname{Pr}_{2}\left(y_{k}^{1}\right)=\operatorname{Pr}_{2}(y)=\operatorname{Pr}_{2}\left(x_{k}^{1}\right), \quad \operatorname{Pr}_{1}\left(x_{k}^{1}\right)<\operatorname{Pr}_{1}(y)<\operatorname{Pr}_{1}\left(y_{k}^{1}\right) \text {. }
$$

Удалим отрезок $\overline{y y_{k}^{1}}$. Пусть $z_{1} \in \mathbf{R}^{2}$ такой, что

$$
\operatorname{Pr}_{2}\left(z^{1}\right)>\operatorname{Pr}_{2}\left(y_{k}^{1}\right), \quad \operatorname{Pr}_{1}\left(z^{1}\right)=\operatorname{Pr}_{1}\left(y_{k}^{1}\right) .
$$

Удалим отрезок $\overline{y_{k-1}^{1} y_{k}^{1}}$ и добавим отрезок $\overline{y_{k-1}^{1} z^{1}}$. Этим способом мы определили укладку $\mu_{1}$ лабиринта $L^{y_{k}^{1}}$ (см. рис. 4в) (вершину $y$ можем сейчас обозначить через $\left.y_{k}^{1}\right)$ и можем предположить, что

$$
\operatorname{diam}\left(\mu^{1}\left(V\left(L^{y_{k}^{1}}\right)\right)\right)<\min \{d(u, v) \mid u, v \in \mu(V(L))\} .
$$




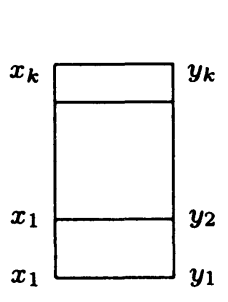

(a)

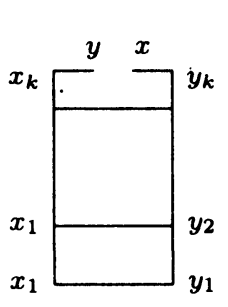

(6)

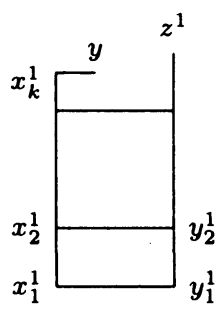

(в)

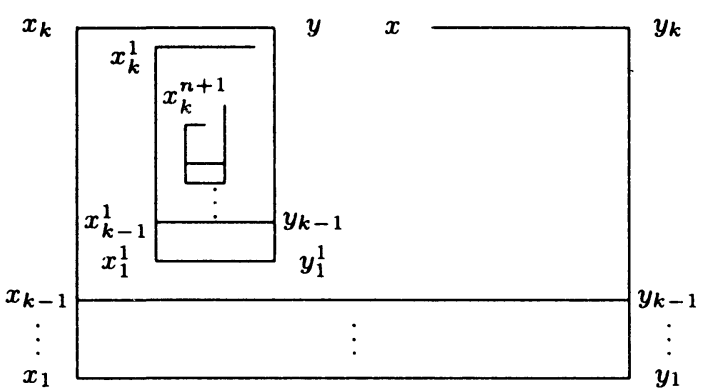

(r)

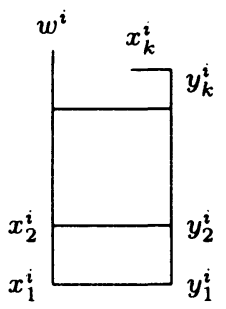

(д)

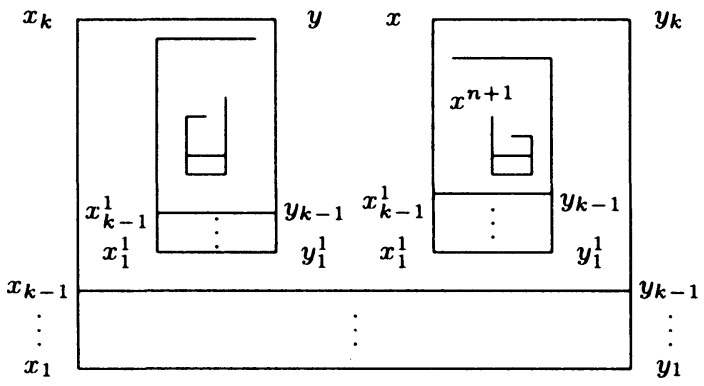

(e)

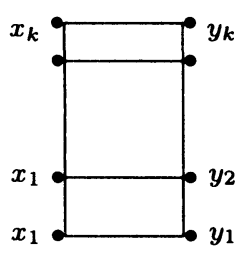

(ж)

Рис. 4.

Сдвигая прямоугольный лабиринт $\overline{L^{y_{k}^{1}}}$ на вектор $\alpha=\overline{z^{1} y}$, получим укладку лабиринта $L^{\prime} \underset{y, z^{1}}{+} L^{y_{k}^{1}}$.

Лабиринт $L^{\dot{y}_{k}^{1}}$ можем вложить в плоскость, как показано на рис. 4в, предполагая, что

$$
\operatorname{diam}\left(V\left(\overline{L^{y_{k}^{2}}}\right)\right)<\operatorname{diam}\left(V\left(\overline{\left(\left(\left(L^{\prime} \underset{y, z^{1}}{+} L^{y_{k}^{1}}\right)_{y_{k}^{1}, z^{2}}^{+} L^{y_{k}^{2}}\right)_{y_{k}^{2}, z^{3}}^{+} \cdots_{y_{k}^{2-1}, z^{i-1}}^{+} L^{y_{k}^{i-1}}\right)}\right)\right)
$$

где $y_{k}^{0}=y, i \in\{2, \ldots, n+1\}$.

Этим способом мы получаем укладку лабиринта $L_{1}$ (см. рис. 4 г).

Лабиринты $L^{x_{k}^{i}}$, изоморфные лабиринту $L^{x}$, можно вложить в плоскость так, 
как показано на рис. 4 д, предполагая, что

$$
\operatorname{diam}\left(V\left(\overline{L^{x_{k}^{2}}}\right)\right) \leqslant \operatorname{diam}\left(V\left(\overline{L^{y_{k}^{1}}}\right)\right) .
$$

Плоская укладка лабиринта $\widetilde{L}$ изображена на рис. 4 е.

Автомат А $q_{0}$ останавливается в лабиринте $\tilde{L}$ и при этом

$$
\operatorname{Tr}\left(\mathbf{A} q_{0} ;\left(L ; v_{0}\right)\right)=\operatorname{Tr}\left(A q_{0} ;\left(\widetilde{L} ; v_{0}\right)\right)
$$

В случае, когда лабиринт $L$ есть $e$-лестница и $\bar{L}$ - одна из его реализаций, поворотом фигуры $\bar{L}$ на угол $\pi / 2$ вокруг любой вершины $x \in V(\bar{L})$ получаем фигуру $\bar{L}^{\prime}$, которая является реализацией $n$-лестницы.

В случае, когда

$$
L=L^{\prime} \underset{x_{1}, x^{2}}{+} L_{1} \underset{x_{2}, x_{2}}{+} \ldots \underset{x_{s}, x^{s}}{+} L_{s}
$$

доказательство аналогично, лишь с той разницей, что при укладывании лабиринта $\widetilde{L}$ дерево $L_{i}$ и ему изоморфные деревья нужно укладывать так, что их диаметры достаточно малы, $1 \leqslant i \leqslant s$. Плоская укладка лабиринта $L$ изображена на рис. 4 ж (лабиринты деревьев $L_{i}, 1 \leqslant i \leqslant s, \varepsilon$-вложимы для каждого $\varepsilon>0$, что изображается символом •.).

\section{3. Доказательство теоремы 2}

Пусть $V=\left\{v_{1}, v_{2}, \ldots, v_{n}\right\}$. Если $p=y_{0}\left(y_{0} y_{1}\right) y_{1}\left(y_{1} y_{2}\right) \ldots y_{m-1}\left(y_{m-1} y_{m}\right) y_{m}$ - маршрут в графе $L=(V, E)$, обозначим через $d(p)$ длину маршрута $p$, а через $[p]_{i}$ - ребро $\left(y_{i-1} y_{i}\right)$ этого маршрута. Пусть

$$
\begin{aligned}
& p_{1}=v_{1}\left(v_{1} v_{i_{1}}^{1}\right) v_{i_{1}}^{1} \ldots v_{i_{s_{1}-1}}^{1}\left(v_{i_{s_{1}-1}}^{1} v_{i_{s_{1}}}^{1}\right) v_{i_{s_{1}}}^{1} \\
& \quad \ldots \\
& p_{n}=v_{n}\left(v_{n} v_{i_{1}}^{n}\right) v_{i_{1}}^{n} \ldots v_{i_{s_{n}-1}}^{n}\left(v_{i_{s_{n}-1}}^{n} v_{i_{s_{n}}}^{n}\right) v_{i_{s_{n}}}^{n}
\end{aligned}
$$

суть маршруты в графе $L=(V, E)$ такие, что

$$
\left\{v_{p}\right\} \bigcup_{j=1}^{s_{p}} v_{i j}^{p}=V, \quad p \in\{1, \ldots, n\}
$$

Пусть $x_{i} \subseteq D, i \in\{1, \ldots, l\}$ такие, что $x_{i} \neq x_{j}$ для $i \neq j, i, j \in\{1, \ldots, l\}$, и для любого $i \in\{1, \ldots, n\}$ существует $j \in\{1, \ldots, l\}$ такое, что $\left[v_{i}\right]_{L}=x_{j}$. Пусть $f: P \rightarrow X$, где $P=\left\{p_{1}, \ldots, p_{n}\right\}$ и $X=\left\{x_{1}, \ldots, x_{l}\right\}$, определено через $f\left(p_{i}\right)=\left[v_{i}\right], i \in\{1, \ldots, n\}$. Обозначим $[f] x_{i}=\left\{p \in P \mid f(p)=x_{i}\right\}$. Пусть $\left|[f]_{x_{i}}\right|=n_{i}$ и $[f] x_{i}=\left\{p_{1}^{i}, \ldots, p_{n_{i}}^{i}\right\}$, $i \in\{1, \ldots, l\}$.

Пусть $\mathbf{A} p_{j}=\left(A, Q_{j}, B, \varphi_{j}, \psi_{j}, q_{1}^{j}\right)$ - инициальный автомат, где

$$
Q j=\left\{q_{1}^{j}, \ldots, q_{s_{j}+1}^{j}, q_{2}^{-j}, \ldots, q_{s_{j}+1}^{-j}\right\} \cup Q_{F}, \quad j \in\{1, \ldots, n\}
$$


такой, что

$$
\begin{aligned}
& \varphi_{j}\left(q_{k}^{j},\left[v_{i_{k-1}}^{j}\right]\right)=q_{k+1}^{j}, \quad \psi_{j}\left(q_{k}^{j},\left[v_{i_{k-1}}^{j}\right]\right)=\left|v_{i_{k-1}}^{j} v_{i_{k}}^{j}\right| \\
& \varphi_{j}\left(q_{k}^{j}, a\right)=q_{k}^{-j}, \quad \psi_{j}\left(q_{k}^{j}, a\right)=\left|v_{i_{k-1}}^{j} v_{i_{k}}^{j}\right|^{-1}, \quad a \neq\left[v_{i_{k-1}}^{j}\right], k \in\left\{2, \ldots, s_{j}\right\}, \\
& \varphi_{j}\left(q_{1}^{j},\left[v_{j}\right]\right)=q_{2}^{j}, \quad \psi_{j}\left(q_{1}^{j},\left[v_{j}\right]\right)=\left|v_{j} v_{i_{1}}^{j}\right| \text {, } \\
& \varphi_{j}\left(q_{1}^{j}, a\right)=q_{F_{0}}, \quad \psi_{j}\left(q_{1}^{j}, a\right)=0, \quad a \neq\left[v_{j}\right], \\
& \varphi_{j}\left(q_{s_{j}+1}^{j},\left[v_{i_{s_{j}}}^{j}\right]\right)=q_{F_{1}}, \quad \psi_{j}\left(q_{s_{j}+1}^{j},\left[v_{i_{s_{j}}}^{j}\right]\right)=0 \text {, } \\
& \varphi_{j}\left(q_{s_{j}+1}^{j}, a\right)=q_{s_{j}+1}^{-j}, \quad \psi_{j}\left(q_{s_{j}+1}^{j}, a\right)=\left|v_{s_{j-1}}^{j} v_{s_{j}}^{j}\right|^{-1}, \quad a \neq\left[v_{i_{j}}^{j}\right] \\
& \varphi_{j}\left(q_{k}^{-j},\left[v_{i_{k-2}}^{j}\right]\right)=q_{k-1}^{-j}, \quad \psi_{j}\left(q_{k}^{-j},\left[v_{i_{k-2}}^{j}\right]\right)=\left|v_{i_{k-3}}^{j} v_{i_{k-2}}^{j}\right|^{-1}, k \in\left\{4, \ldots, s_{j}+1\right\} \text {, } \\
& \varphi_{j}\left(q_{3}^{-j},\left[v_{i_{1}}^{j}\right]\right)=q_{2}^{-j}, \quad \psi_{j}\left(q_{3}^{-j},\left[v_{i_{1}}^{j}\right]\right)=\left|v_{i_{1}}^{j} v_{j}\right|^{-1}, \\
& \varphi_{j}\left(q_{2}^{-j}, a\right)=q_{F_{0}}, \quad \psi_{j}\left(q_{2}^{-j}, a\right)=0 .
\end{aligned}
$$

Заметим, что автомат $\mathbf{A} p_{j}$ распознает лабиринт $L v_{j}=\left(V ; v_{j}\right)$, и при этом, если мозаичный лабиринт $L_{v}, L_{v} \neq L v_{j}$, то в обходе лабиринта $L_{v}$ автомат $\mathbf{A} p_{j}$ вернется в вершину $v, j \in\{1, \ldots, n\}$. Через $q_{i}\left(\mathbf{A} p_{j}\right)$ обозначим состояние $q_{|i|}^{\operatorname{sign}(i) j}$ автомата

$$
\mathbf{A} p_{j}, i \in\left\{1, \ldots, s_{j}+1,-2, \ldots,-\left(s_{j}+1\right)\right\}
$$

(это обозначение используем и для состояния автомата $\mathbf{A} q_{0} ;$ автомат $\mathbf{A} q_{0}$ определим ниже).

Определим автомат $\mathbf{A} q_{0}=\left(A, Q, B, \varphi, \psi, q_{0}\right)$, где

$$
Q=\left\{q_{0}\right\} \bigcup_{j=1}^{k} Q_{j} \backslash\left\{q_{1}^{j}\right\}
$$

следующим образом.

Пусть $m \in\{1, \ldots, l\}$,

$$
\begin{gathered}
\varphi\left(q_{0}, x_{m}\right)=q_{2}\left(\mathbf{A} p_{1}^{m}\right), \quad \psi\left(q_{0}, x_{m}\right)=\left|\left[p_{1}^{m}\right]_{1}\right|_{L}, \\
\varphi\left(q_{0}, a\right)=q_{F_{0}}, \quad \psi\left(q_{0}, a\right)=0, \quad a \notin X, \\
\varphi\left(q_{-2}\left(\mathbf{A} p_{i}^{m}\right), x_{m}\right)=q_{2}\left(\mathbf{A} p_{i+1}^{m}\right), \quad \psi\left(q_{0}, x_{m}\right)=\left|\left[p_{i+1}^{m}\right]_{1}\right|_{L}, \quad i \in\left\{1, \ldots, n_{m}-1\right\} \\
\varphi\left(q_{-2}\left(\mathbf{A} p_{n_{m}}^{m}\right), x_{m}\right)=\varphi_{b}\left(q_{-2}\left(\mathbf{A} p_{n_{m}}^{m}\right), x_{m}\right), \quad \psi\left(q_{-2}\left(\mathbf{A} p_{n_{m}}^{m}\right), x_{m}\right)=\psi_{b}\left(q_{-2}(\mathbf{A}), x_{m}\right),
\end{gathered}
$$

где $b$ такое, что $p_{b}=p_{n_{m}}^{m}$,

$$
\varphi\left(q_{j}^{i}, a\right)=\varphi_{i}\left(q_{j}\left(\mathbf{A} p_{i}\right), a\right), \quad \psi\left(q_{j}^{i}, a\right)=\psi_{i}\left(q_{j}\left(\mathbf{A} p_{i}\right), a\right),
$$

для $j \in\left\{2, \ldots, s_{i}+1,-3, \ldots,-\left(s_{i}+1\right)\right\}, i \in\{1, \ldots, n\}$.

\section{4. Доказательство теоремы 3}

Пусть $z \in K \subseteq \mathbf{Z}^{2}$. Положим

$$
z_{(i, j)}= \begin{cases}1, & z+(i, j) \in K \\ 0, & z+(i, j) \notin K\end{cases}
$$


$i, j \in\{0,1,-1\}$. Ясно что $z_{(0,0)}=1$. Пусть

$$
O(z)=\left(z_{(1,-1)}, z_{(1,0)}, z_{(1,1)}, z_{(0,-1)}, z_{(0,1)}, z_{(-1,-1)}, z_{(-1,0)}, z_{(-1,1)}\right) \in\{0,1\}^{8}
$$

- окрестность точки $z \in K$ и

$$
\mathbf{O}(z)=\left\{z+(i, j) \mid z_{(i, j)}=1, \quad i, j \in\{0,1,-1\}\right\}
$$

Пусть

$$
a=\left\{w_{1}, \ldots, w_{p}\right\} \subseteq D, \quad 1 \leqslant p \leqslant 4, \quad \lambda \in N, \quad \lambda>2 .
$$

Через $L\left(a, x_{0}, \lambda\right)$ обозначим следующее семейство инициальных $\pi$-лабиринтов $L=$ $\left(V(L), x_{0}\right)$, где

$$
V(L)=\left[x_{0}, x_{0}+\lambda w_{1}\right] \cup \ldots \cup\left[x_{0}, x_{0}+\lambda w_{p}\right] \cup O\left(x_{0}+\lambda w_{1}\right) \cup \ldots \cup O\left(x_{0}+\lambda w_{p}\right),
$$

и $O\left(x_{0}+\lambda w_{i}\right)$ такие, что

$$
\begin{aligned}
& \text { если } w_{i}=e, \text { то } O\left(x_{0}+\lambda w_{i}\right)=(*, *, *, *, *, 0,1,0), \\
& \text { если } w_{i}=n \text {, то } O\left(x_{0}+\lambda w_{i}\right)=(0, *, *, 1, *, 0, *, *), \\
& \text { если } w_{i}=w \text {, то } O\left(x_{0}+\lambda w_{i}\right)=(0,1,0, *, *, *, *, *), \\
& \text { если } w_{i}=s \text {, то } O\left(x_{0}+\lambda w_{i}\right)=(*, *, 0, *, 1, *, *, 0), \text { где } * \in\{0,1\} .
\end{aligned}
$$

Точки $x_{0}+j w_{i}$ обозначим через $w_{i}^{j}, j \in\{0,1, \ldots, \lambda\}, i \in\{1, \ldots, p\}$. Через $L\left(a, x_{0}, \lambda, O_{1}, \ldots, O_{p}\right)$ обозначим инициальный $\pi$-лабиринт из семейства $L\left(a, x_{0}, \lambda\right)$, в котором известны окрестности концевых точек $O_{i}=O\left(w_{i}^{\lambda}\right), i \in\{1, \ldots, p\}$. Через $V(a)$ обозначим множество $\left\{x_{0}, w_{1}^{\lambda}, \ldots, w_{p}^{\lambda}\right\}$.

Лемма 1. Пусть $\mathbf{A}=(A, Q, B, \varphi, \psi)-$ произвольнал пешка, $\|Q\| \geqslant 3$. Тогда для любых $q \in Q, a=\left\{w_{1}, \ldots, w_{p}\right\} \subseteq D, n, m \in N$, если $\lambda=\|Q\|$ !, то в случае, когда $\operatorname{st}\left(\pi^{\prime}, V(a)\right)$ определенно,

$$
\operatorname{st}(\pi, V(a))=\operatorname{st}\left(\pi^{\prime}, V(a)\right), \quad \operatorname{pl}\left(\pi^{\prime}, V(a)\right)=\operatorname{pl}(\pi, V(a)),
$$

əде

$$
\pi=\pi\left(\mathbf{A}, L\left(a, x_{0}, n \lambda, O_{1}, \ldots, O_{p}\right), \quad \pi^{\prime}=\pi\left(\mathbf{A}, L\left(a, x_{0}, m \lambda, O_{1}, \ldots, O_{p}\right)\right.\right.
$$

Доказательство. Достаточно показать, что утверждение леммы имеет место для $m=1$.

Если $\operatorname{pl}\left(\pi^{\prime}, V(a)\right)=x_{0}$, то достаточно рассмотреть два случая: автомат $A$ не находился в точке $w_{s}^{\lambda-1}$ и автомат $A$ находился в точке $w_{s}^{\lambda-1}$ для некоторого $s \in$ $\{1, \ldots, p\}$.

В первом случае ясно, что $\operatorname{pl}(\pi, V(a))=x_{0}$ и $s t(\pi, V(a))=\operatorname{st}\left(\pi^{\prime}, V(a)\right)$. Рассмотрим второй случай. Пусть

$$
\begin{aligned}
q_{1}^{k^{\prime}}=\operatorname{st}\left(\pi^{\prime}, w_{s}^{k^{\prime}}\right), & 0 \leqslant k^{\prime} \leqslant \lambda-1, \\
q_{2}^{k^{\prime \prime}}=\operatorname{st}\left(\pi, w_{s}^{k^{\prime \prime}}\right), & 0 \leqslant k^{\prime \prime} \leqslant n \lambda-1
\end{aligned}
$$


(пока мы не можем утверждать, что значения $q_{2}^{k^{\prime \prime}}, 1 \leqslant k^{\prime \prime} \leqslant n \lambda-1$, определены, но из последующего это будет ясно).

Ясно, что $q_{1}^{k}=q_{2}^{k}$, для $0 \leqslant k \leqslant \lambda-1$. Из того, что $\lambda-2>\|Q\|$, следует, что $q_{1}^{\lambda-1}=q_{1}^{k}$ для некоторого $k \in\{2, \ldots, \lambda-2\}$. Отсюда, $q_{2}^{k^{\prime \prime}}$ определено, $\lambda-1<k^{\prime \prime}<n \lambda$. Пусть $k_{\max }=\max \left\{k \in\{2, \ldots, \lambda-2\} \mid q_{1}^{\lambda-1}=q_{1}^{k}\right\}$. Тогда $\lambda-1-k_{\max } \leqslant\|Q\|$ и при этом $q_{2}^{\lambda-1}=q_{2}^{\lambda-1+j\left(\lambda-1-k_{\max }\right)}$ для всех $j \in \mathbf{Z}^{+}$таких, что $\lambda-1+j\left(\lambda-1-k_{\max }\right)<n \lambda$. Последнее имеет место и для $j=\lambda(n-1) /\left(\lambda-1-k_{\max }\right)$, и мы получаем, что $q_{1}^{\lambda-1}=q_{2}^{\lambda-1}=q_{2}^{n \lambda-1}$. Поэтому автомат А будет в том же самом состоянии $q=q_{1}^{\lambda-1}$ и в вершине $w_{s}^{n \lambda-1}$. Но $O\left(w_{s}^{\lambda-1}\right)=O\left(w_{s}^{\lambda n-1}\right)$, то есть автомат А на входе имеет тот же самый символ. Из равенства $\operatorname{pl}\left(\pi^{\prime}, V(a)\right)=x_{0}$, следует, что автомат А вернется в вершину $x_{0}$. При этом возвращении в точку $x_{0}$ автомат $\mathbf{A}$ пройдет вершину $w_{s}^{1}$. Так же получается, что автомат $\mathbf{A}$ в точках $\left(w_{s}^{1}\right)$ будет в этом же состоянии. Из того, что $\lambda-2>\|Q\|$, следует, что $q_{1}^{1}=q_{1}^{k}$ для некоторого $k \in\{2, \ldots, \lambda-2\}$.) Пусть $k_{\min }=\min \left\{k \in\{2, \ldots, \lambda-2\} \mid q_{1}^{1}=q_{1}^{k}\right\}$. Тогда $\lambda-1-k_{\min } \leqslant\|Q\|$ и при этом $q_{2}^{n \lambda-1-i}=q_{1}^{\lambda-1-i}$ для $i>0$, такого, что $\lambda-1-i>1$. Для $\lambda-1-i=k_{\min }$ получаем, что $q_{2}^{(n-1) \lambda+k_{\min }}=q_{1}^{k_{\min }}$. Тогда $q_{2}^{(n-1) \lambda+k_{\min }}=q_{2}^{(n-1) \lambda+k_{\min }-s\left(k_{\min }-1\right)}$ для каждого $s$ такого, что $2<(n-1) \lambda+k_{\min }-s\left(k_{\min }-1\right)$. Для $s=(n-1) \lambda /\left(k_{\min }-1\right)$ получаем, что $q_{2}^{k_{\min }}=q_{1}^{k_{\min }}$. Тогда $q_{2}^{k_{\min }-j}=q_{1}^{k_{\min }-j}$ для $\left.0<j<k_{\min }-1\right)$.

Если $\operatorname{pl}\left(\pi^{\prime}, V(a)\right)=w_{s}^{\lambda}$, то автомат был в вершине $w_{s}^{\lambda-1}$ (уже показано, что автомат А будет в том же самом состоянии и в вершине $w_{s}^{n \lambda-1}$ и на входе будет иметь тот же самый символ), так что

$$
\operatorname{pl}(\pi, V(a))=w_{s}^{\lambda n}, \quad \operatorname{st}(\pi, V(a))=\operatorname{st}\left(\pi^{\prime}, V(a)\right) .
$$

Предположим, что существует пешка $\mathbf{A}_{0}=\left(A, Q_{0}, B, \varphi_{0}, \psi_{0}, q_{0}\right)$, которая распознает класс $\left(\mathbf{C}_{0}, \mathrm{H \Pi}\right)$. Ясно, что $\left\|Q_{0}\right\|>2$.

Рассмотрим подкласс $\mathbf{C}_{0}^{\prime}$ класса $\mathbf{C}_{0}$, который будет определен ниже.

Пусть $z_{i}=\left(x_{i}, y_{i}\right) \in \mathbf{Z}^{2}, i \in\{1,2\}$, такие, что

$$
x_{2}<x_{1}-1, \quad y_{2}>y_{1}+1,
$$

и

$$
K_{0}^{\left\{z_{i}\right\}_{i=1,2}}=\left\{x_{1}\right\} \times\left[y_{1}, y_{2}\right] \cup\left\{x_{2}\right\} \times\left[y_{1}, y_{2}\right] \cup\left[x_{2}, x_{1}\right] \times\left\{y_{1}\right\} \cup\left[x_{2}, x_{1}\right] \times\left\{y_{2}\right\}
$$

Тогда

$$
\begin{aligned}
\mathbf{C}_{0}^{\prime}=\left\{c: Z^{2} \rightarrow E^{2} \mid\right. & c^{-1}(\{1\})=K_{0}^{\left\{z_{i}\right\}_{i=1,2}}, \\
& \text { где } \left.z_{1}, z_{2} \in Z^{2} \text { удовлетворяют указанному выше условию }\right\}
\end{aligned}
$$

Определим лабиринт $L_{0}=\left(V_{0}, E_{0}\right)$, полагая

$$
\begin{gathered}
V_{0}=\left\{v_{0}, v_{1}, v_{2}, v_{3}\right\}, \quad E_{0}=\left\{\left\langle v_{0}, v_{1}\right\rangle,\left\langle v_{1}, v_{2}\right\rangle,\left\langle v_{2}, v_{3}\right\rangle,\left\langle v_{3}, v_{0}\right\rangle\right\} \\
\left|\left(v_{0}, v_{1}\right)\right|=w, \quad\left|\left(v_{1}, v_{2}\right)\right|=n, \quad\left|\left(v_{2}, v_{3}\right)\right|=e, \quad\left|\left(v_{3}, v_{0}\right)\right|=s .
\end{gathered}
$$

Ясно, что $L_{0}$ есть $n$-лестница (то есть $e$-лестница).

Пусть лабиринт $\widetilde{L}_{0}$ получен из лабиринта $L_{0}$ так, как в теореме 1 , где $n=\left\|Q_{0}\right\|$. Выберем укладку этого лабиринта так же, как и в теореме 1. Из этой укладки можем 
получить укладку, у которой вершины расположены в узлах подрешетки целочисленной решетки с шагом $\lambda_{0}=\left\|Q_{0}\right\|$ ! [4]. Рассмотрим так полученный мозаичный лабиринт $L_{0}^{\prime}$ и поведение пешки $\mathbf{A}_{0}$ в нем. Из леммы 1 , из геометрии лабиринта $L_{0}^{\prime}$, и из того, что если автомат распознает лабиринт, то в одной вершине автомат находится не больше, чем $\left\|Q_{0}\right\|$ раз, вытекает, что автомат $\mathbf{A}_{0}$ останавливается в состоянии $q_{F_{1}}$. Теорема доказана.

\section{Список литературы}

1. Килибарда Г., Новое доказательство теоремы Будаха-Подколзина. Дискретная математиха (1991) 3, №3, 135-146.

2. Кудрявцев В. Б., Подколзин А. С., Ушчумлич Ш., Введение в теорию абстрахтнъх автоматов. Наука, Москва, 1985.

3. Кудрявцев В. Б., Алешин С. В., Подколзин А. С., Введение в теорию автоматов. Наука, Москва, 1985.

4. Харари Ф., Теория графов. Мир, Москва, 1973.

Статья поступила 17.05.1999. 